\title{
1 A unique small molecule pair controls the plant circadian clock
}

2

3 Takahiro N Uehara ${ }^{1}$, Saori Takao ${ }^{2}$, Hiromi Matsuo ${ }^{2}$, Ami N. Saito ${ }^{3}$, Eisuke Ota ${ }^{3}$, Azusa

$4 \mathrm{Ono}^{4}$, Kenichiro Itami ${ }^{1,2,5}$, Toshinori Kinoshita ${ }^{2,4}$, Junichiro Yamaguchi ${ }^{3, *}$, Norihito

$5 \quad$ Nakamichi $^{2,4, *}$.

6

7 1. Dept. Chem., Grad. Sch. Sci., Nagoya Univ., Furo-cho, Chikusa, Nagoya 464-8602,

8 Japan, 2. Inst. Transformative Bio-Molecules (WPI-ITbM), Nagoya University, Furo-cho,

9 Chikusa, Nagoya 464-8601, Japan, 3. Dept. Appl. Chem., Waseda Univ., Ohkubo 3-4-1,

10 Shinjuku, Tokyo 169-8555, Japan. 4. Div. Biol. Sci., Grad. Sch. Sci., Nagoya University,

11 Furo-cho, Chikusa, Nagoya 464-8602, Japan, 5. JST ERATO, Itami Molecular

12 Nanocarbon Project, Nagoya Univ., Furo-cho, Chikusa, Nagoya 464-8602, Japan,

$13 *$ To whom correspondence should be addressed. E-mail: nnakamichi@itbm.nagoya-

14 u.ac.jp, +81-52-789-3656 (N.N); junyamaguchi@waseda.jp (J.Y.)

15

16 Total word count (Introduction, Material and Methods, Results, Discussion): 3,259

17 Word counts for Introduction : 1,172

18 Word counts for Material and Methods : 343

19 Word counts for Results : 1,257

20 Word counts for Discussion : 487

21

22 Number of Figures : 5

23 Number of Color Figures : 5

24 Number of Tables : 0

25 Supporting Information : Method of synthesis of chemical compounds, 2 supporting

26 figures 


\section{Summary}

Circadian clocks are the biological time keeping systems that coordinate genetic, metabolic, and physiological behaviors with the external day-night cycle. Previous studies have suggested possible molecular mechanisms for the circadian clock in Arabidopsis thaliana (Arabidopsis), but there might be additional mechanisms that have been hidden due to genetic redundancy.

A clock reporter line of Arabidopsis was screened against the 10,000 chemicals

in the Maybridge Hitfinder10K chemical library, and a structure-activity relationship study of hit compounds was conducted. Clock mutants were treated with two of the small molecules to gain insight into their mode of action. pyrazolo[3,4- $d]$ pyrimidine-4,6(5H)-dione (TU-892) as a period lengthening molecule. From a structure-activity relationship study, we found that a molecule possessing 2,4dichlorophenyl instead of a 3,4-dichlorophenyl group (TU-923) had period shortening activity. The period shortening activity of $\mathbf{T U} \mathbf{- 9 2 3}$ was reversed to a lengthening activity in double mutants lacking PSEUDO-RESPONSE REGULATOR 9 (PRR9) and PRR7 (prr9-10 prr7-11).

Our study provides a unique small molecule pair that regulates the pace of the clock in opposite ways, likely by targeting unknown factors. Small differences at the atomic level can reverse the period tuning activities. $P R R 9$ and $P R R 7$ are essential for the activity of TU-923 in period shortening. 
bioRxiv preprint doi: https://doi.org/10.1101/2020.05.25.113746; this version posted May $27,2020$. The copyright holder for this preprint (which was not certified by peer review) is the author/funder, who has granted bioRxiv a license to display the preprint in perpetuity. It is made available under aCC-BY-ND 4.0 International license.

49 Key words: Arabidopsis thaliana, circadian clock, high-throughput chemical screening,

50 small molecules, structure-activity relationship (SAR) 


\section{Introduction}

52 Circadian clocks are the biological timekeeping systems that govern the approximately

53 24-h rhythms of genetic, metabolic, physiological, and behavioral processes in most

54 organisms. This oscillation allows organisms to predict and anticipate day-night changes

55 in the environment. For instance, at dawn Arabidopsis thaliana (Arabidopsis) induces the

56 expression of genes in the phenylpropanoid pathway that produce secondary metabolites

57 capable of acting as phenolic sunscreens by absorbing ultraviolet and short-wavelength

58 visible light (Harmer et al., 2000). This phenomenon seems to be important for plants to

59 anticipate and adapt to sunrise, which otherwise could result in photodamage to

60 chloroplasts (Harmer et al., 2000). Arabidopsis induces jasmonate defense hormones

61 during the day for protection against damage from a herbivorous caterpillar, Trichoplusia

$62 n i$, whose feeding activity is higher during the daytime and maximal at dusk (Goodspeed

63 et al., 2012). The plant clock contributes to fitness in $24 \mathrm{~h}$ day-night cycles even under

64 laboratory conditions by coordinating the rhythms of many biological processes (Dodd et

65 al., 2005; Yerushalmi et al., 2011). In addition to day-night anticipation, the clock is used

66 to measure photoperiod for inducing flower meristem formation in many plants

67 (Yanovsky \& Kay, 2002). Consistently, genetic mutations in the clock are often found in

68 some crop cultivars whose flowering times have changed due to human selection, thereby

69 allowing the spread of these cultivars into areas or regions where the latitude or climate

70 is different from the regions of origin for these crop plants (Nakamichi, 2015). 
73 McClung, 2019). Evidence from genetic and biochemical studies have been taken to

74 suggest the presence of Arabidopsis TTFLs. Among the TTFLs, there are some

75 genetically redundant clock-associated genes (Millar, 2016; Nohales \& Kay, 2016;

76 McClung, 2019) due to whole-genome duplication followed by local duplication during

77 evolution (Arabidopsis Genome Initiative, 2000). To investigate molecular mechanisms

78 that potentially involve genetic redundancy, chemical genetic approaches have emerged.

79 Chemical compounds used in these studies also overcome the lethality of gene mutations

80 for studying gene function. Chemical compounds can also be applied in a dose-dependent,

81 time-dependent, or growth stage-conditional manner, allowing stringent controls to be

82 employed for each of the biological processes of interest (Uehara et al., 2019).

83 Several studies have demonstrated that control of the animal clock can be

84 achieved by small molecules, which will ultimately provide clock-modulator-based

85 medicines (Hirota et al., 2008; Isojima et al., 2009; Hirota et al., 2010; Hirota et al., 2012;

86 Tamai et al., 2018; Oshima et al., 2019). In plants, some natural or synthetic small

87 molecules that modulate the clock have been reported. Endogenous sugars can entrain the

88 Arabidopsis circadian clock (Haydon et al., 2013). Prieurianin, also known as endosidin

89 1, was found to be a period shortening natural compound that perturbs actin cytoskeleton

90 dynamics in Arabidopsis (Toth et al., 2012). Molecules capable of destabilizing

91 (latrunculin B and cytochalasin D) or stabilizing (jasplakinolide) actin also shorten the

92 Arabidopsis circadian period (Toth et al., 2012). Tetraethylammonium, a $\mathrm{K}^{+}$channel

93 blocker, shortens the circadian period in duckweed (Lemna gibba G3) (Kondo, 1990).

94 Brevicompanine, a naturally occurring fungal compound, alters the expression of some 
95 clock-associated genes in Arabidopsis (de Montaigu et al., 2017). Trichostatin A, a

96 histone deacetylase inhibitor, causes a phase delay in clock-associated genes expressed

97 during the evening in Arabidopsis (Perales \& Mas, 2007).

99 found synthetic small molecules that lengthen the Arabidopsis circadian clock (Ono et al.,

100 2019; Uehara et al., 2019). We have focused on molecules that change the circadian

101 period since the robustness of period length stability in the midst of environmental

102 fluctuations, such as temperature changes, is an important feature of circadian clocks.

103 This property is striking from the point of view of the Arrhenius equation that describes

104 the temperature dependence of chemical reactions (chemical reaction rates increase if

105 temperatures increase). Generally, a $10^{\circ} \mathrm{C}$ increase causes a 2-fold increase in reaction

106 rate $\left(\mathrm{Q}_{10}=2\right)$. A chemical oscillation, the Belousov-Zhabotinsky reaction, follows the

107 Arrhenius equation, and the period of oscillation is greatly shortened by increased

108 temperatures (Bansagi et al., 2009). The period of plant circadian clocks is not

109 significantly altered by temperature changes, and the $\mathrm{Q}_{10}$ is far less than 2; this is called

110 the temperature compensation of the period length (Salome et al., 2010). Temperature

111 compensation of circadian period length is found in the phosphorylation-

112 dephosphorylation cycle of cyanobacterial KaiC clock proteins even in vitro (Nakajima

113 et al., 2005). If essential clock components are mutated, periods change duration. For

114 example, both short- and long-period mutants were found for a Drosophila period gene

115 (Bargiello et al., 1984). Mutations in cyanobacterial KaiC resulted in short, long, or

116 arrhythmic phenotypes, depending on the site of mutation (Ishiura et al., 1998). Tuning 
117 the expression level of two clock-associated genes, TIMING OF CAB EXPRESSION 1

118 (TOC1) or ZEITLUPE (ZTL), changes the period length, suggesting that period length is

119 controlled by these genes (Mas et al., 2003; Somers et al., 2004). Thus, period change is

120 a sign of perturbation or disorder of the clock.

121

PHA767491 was found to be a period lengthening molecule from the LOPAC

122 library (Library of Pharmacologically Active Compounds that modulate a broad range of

123 biological processes in mammals and microorganisms) that inhibited the casein kinase 1

124 family proteins in Arabidopsis (CKL proteins) (Uehara et al., 2019). Through

125 derivatization of PHA767491, we developed more a potent and selective Arabidopsis

126 CKL inhibitor, AMI-331, that lengthens the period at higher nanomolar levels (Saito et

127 al., 2019). 3,4-Dibromo-7-azaindole (B-AZ) was also found as a period lengthening

128 molecule from our in-house 'ITbM' chemical library that is enriched in plant hormone

129 mimicking molecules, and B-AZ also inhibits CKLs (Ono et al., 2019). These studies

130 further found that treatment with these CKL inhibitors results in the accumulation of

131 PSEUDO-RESPONSE REGULATOR 5 (PRR5) and TOC1 (known as PRR1) proteins,

132 two transcriptional repressors in the clock genetic circuit. Consistently, CKL4 directly

133 phosphorylates PRR5 and TOC1 for degradation (Uehara et al., 2019). Finding a small

134 molecule that modulates the circadian clock, followed by revealing the action mechanism

135 of the hit molecule, makes it possible to reveal the molecular mechanisms underlying the

136 clock. Therefore, chemical biology focusing on the plant clock provides considerable

137 knowledge and chemical tools for developing and optimizing agrochemical use (de

138 Montaigu et al., 2017; Belbin et al., 2019; Panter et al., 2019; Uehara et al., 2019). 
140 report here a high-throughput phenotypic screening using a different chemical library, the

141 Maybridge Hitfinder 10K. 5-(3,4-Dichlorophenyl)-1-phenyl-1H-pyrazolo[3,4-d]

142 pyrimidine-4,6(5H,7H)-dione (TU-892) was found to be a period lengthening molecule.

143 A structure-activity relationship (SAR) study indicated that both the pyrazole ring and the

144 pyrimidinedione moiety are essential. A molecule substituting a 2,4-dichlorophenyl

145 moiety instead of a 3,4-dichlorophenyl group (TU-923), showed period-shortening

146 activity. Our study provides evidence that a small difference at the atomic level can

147 control the clock in opposite ways. Our results also suggest that there are crucial unknown

148 factors involved in the clock mechanism that remain to be identified.

\section{$150 \quad$ Materials and methods}

\section{Screening of small molecules that change the circadian period}

152 Seeds of Arabidopsis thaliana accession Col-0 harboring CCA1:LUC (Nakamichi et al.,

153 2005) (a reporter construct that expresses luciferase with peak expression in the early

154 morning) were sterilized and placed on half-strength Murashige-Skoog (MS) plates

155 containing $0.25 \%(\mathrm{w} / \mathrm{v})$ sucrose. Seeds were kept at $4^{\circ} \mathrm{C}$ in the dark for 2 days followed

156 by transfer to $22^{\circ} \mathrm{C}$ with $12 \mathrm{~h}$ light $\left(\sim 70 \mu \mathrm{mol} \mathrm{s}^{-1} \mathrm{~m}^{-2}\right) / 12 \mathrm{~h}$ dark conditions (LD). Four

157 days after incubation, young seedlings were individually transferred with a dropper to a

158 well of a 96-well plate. Seedlings were treated with small molecules from the Maybridge

159 Hitfinder $10 \mathrm{~K}$ library at a final concentration of $50 \mu \mathrm{M}$ and $500 \mu \mathrm{M}$ luciferin (120-05114,

160 Wako). After an additional one-day incubation in LD conditions, the plates were read by 
161 a CL96 time-lapse luminescence detector (Churitsu, Toyoake, Japan). The circadian

162 rhythm of the luminescent reporter was calculated as previously reported (Kamioka et al.,

163 2016). The molecule that changed the circadian period, [5-(3,4-dichlorophenyl)-1-

164 phenyl-1H-pyrazolo[3,4- $d]$ pyrimidine-4,6(5H,7H)-dione $\quad(\mathbf{T U}-892)], \quad$ was further

165 confirmed by testing with different concentrations of the small molecule using

166 CCA1:LUC and the evening reporter construct TOC1:LUC (Uehara et al., 2019).

167

168 In vitro phosphorylation assays of Arabidopsis CKLs

169 In vitro phosphorylation assays of Arabidopsis CKL4 and CKL1 were performed as 170 previously reported (Uehara et al., 2019). PHA767491 was purchased from Sigma

171 Chemical Corp., dissolved in DMSO at a concentration of $10 \mathrm{mM}$, and stored at $-20^{\circ} \mathrm{C}$ 172 until use.

173

174 Synthesis of TU-892 analogues

175 Synthesis of TU-892 analogues is described in Supporting Information-Methods. TU-

$176 \mathbf{8 9 2}$ analogues were dissolved in DMSO at a concentration of $10 \mathrm{mM}$ and stored at room

177 temperature. The effect of TU-892 analogues on the circadian period length of

178 Arabidopsis seedlings was tested as described above.

180 TU-892 and TU-923 treatments of clock-period mutants

181 Clock-period mutants, ccal-1 lhy-12 CCA1:LUC (Kamioka et al., 2016), prr9-10 prr7-

18211 CCA1:LUC (Nakamichi et al., 2005), prr7-11 prr5-11 CCA1:LUC (Nakamichi et al., 
183 2005), and prr5-11 toc1-2 CCA1:LUC (Uehara et al., 2019) were treated with TU-892

184 and TU-923 and the circadian rhythm assay was performed as described above.

185

186 Results

187

188 Screening synthetic small molecules that change the circadian period

189 To find small molecules capable of changing the circadian period of Arabidopsis

190 seedlings, we conducted a high-throughput phenotypic screening using Maybridge

191 Hitfinder 10K, a chemical library that is different from those used in our previous studies

192 (Ono et al., 2019; Uehara et al., 2019). We monitored the circadian rhythm of the

193 luminescent luciferase reporter driven by the CIRCADIAN CLOCK-ASSOCIATED 1

194 (CCA1) promoter (CCA1:luciferase [LUC]), whose expression peaks in the early morning

195 (Fig. 1a). 5-(3,4-Dichlorophenyl)-1- phenyl-1H-pyrazolo[3,4- $d]$ pyrimidine-4,6(5H,7H)-

196 dione (TU-892) was found to be a period lengthening molecule (Fig. 1b). TU-892

197 lengthened the period of not only CCA1:LUC but also TIMING OF CAB EXPRESSION

198 1:LUC (TOC1:LUC), whose luminescence peaks in the evening (Uehara et al., 2019) in

199 a dose-dependent fashion (Fig. 1c,d,e). These results established TU-892 as a period-

200 lengthening molecule for the Arabidopsis circadian clock. Lower concentrations $(25 \mu \mathrm{M})$

201 of TU-892 lengthen the circadian period about 2 h. Another parameter of the clock,

202 amplitude, was not evaluated precisely in our test as previously reported (Ono et al.,

203 2019). Because we carefully selected seedlings of similar size, seedling size was unlikely

204 the reason for the variable amplitude. We hypothesize that other factors, such as 
205 fluctuations in temperature during the assay, might have affected the amplitude. Variation

206 in amplitude responding to environment fluctuations such as temperature is a general 207 aspect of circadian rhythms (Murayama et al., 2017). We also noticed that treatment with $208100 \mu \mathrm{M}$ TU-892 caused bleaching of the seedlings, an indication of toxicity of this 209 molecule at higher concentrations (Fig. S1). Since alterations of the circadian clock by 210 genetic mutations do not cause lethality, $\mathbf{T U}-892$ at higher concentrations affects not only

211 the circadian clock, but also essential physiological processes. Collectively, our result 212 show that TU-892 modulates an indispensable property of the clock, that is, the 213 robustness of period length.

\section{TU-892 is not a CKL inhibitor}

216 Given that three other period lengthening molecules (PHA767491, AMI-331, and B-AZ)

217 inhibit CKL kinase activity (Ono et al., 2019; Saito et al., 2019; Uehara et al., 2019), we 218 examined whether TU-892 also inhibits CKL. The CKL4 kinase activity for the model 219 substrate casein was tested, because CKL4 kinase activity was the strongest among the 220 purified CKL proteins (Uehara et al., 2019). PHA767491 at concentrations of 4 to 100 $221 \mu \mathrm{M}$ strongly inhibited CKL4 kinase activity as previously reported (Uehara et al., 2019), 222 whereas the same concentration of TU-892 did not inhibit CKL4 kinase activity at all 223 (Fig. 2a). PHA767491 also inhibited CKL1 kinase activity, whereas TU-892 did not (Fig. $224 \mathbf{2 b}$ ), showing that $\mathbf{T U}-\mathbf{8 9 2}$ is not a CKL inhibitor.

225 We also examined the interaction between CK1 inhibition and TU-892 for period 226 lengthening (Fig. 2c,d). PHA767491 treatment at a concentration of $250 \mu \mathrm{M}$ lengthened 
227 the period by about $3 \mathrm{~h}$, as previously reported (Uehara et al., 2019). At a concentration 228 of $250 \mu \mathrm{M}$, the effect of PHA767491 on the lengthening period is mostly saturated. If the 229 mode of action of TU-892 is dependent on CK1, TU-892 should not lengthen the period 230 in Arabidopsis treated with $250 \mu \mathrm{M}$ PHA767491. Our results showed that TU-892 231 lengthened the period in seedlings treated with $250 \mu \mathrm{M}$ PHA767491 in a dose-dependent 232 manner. This result suggested that TU-892 lengthens the period independent of the 233 inhibition of CK1 activity. The structure of $\mathbf{T U - 8 9 2}$ is not similar to other molecules that 234 potentially modulate clock parameters such as sugars, prieurianin, latrunculin B, 235 cytochalasin D, jasplakinolide, tetraethylammonium, brevicompanine, and trichostatin A.

\section{Structure-activity relationship study of $\mathbf{T U}-892$}

238 To gain insight into the molecular mode of action of TU-892 (1a) on period lengthening, 239 we performed a structure-activity relationship (SAR) study. We initially synthesized TU240892 and analogues as described in Supporting Information-Methods. We first tested 241 whether the pyrimidinedione moiety is essential for period-lengthening activity (Fig. 3a).

242 Methyl substitution of pyrimidinedione had no period-lengthening activity (2). 243 Replacement of the pyrimidinedione with a benzene ring had no activity, too (3). These 244 results indicated that the pyrimidinedione is essential for period lengthening activity. Next, 245 we evaluated the importance of the pyrazole ring. Two molecules replacing pyrazole with 246 thiophene had quite weak or no period-lengthening activity (4a,b, Fig. $\mathbf{3 b})$. Note that 247 these molecules lack the phenyl group on the pyrazole, suggesting that either the pyrazole 248 or the phenyl group is essential for activity. Next, we substituted the phenyl group on the 
252 (19), or $o$-bromophenyl (1h) group resulted in the loss of period-lengthening activity (Fig.

253 3c). Replacement of the phenyl group with $p$-methoxyphenyl (1d) retained the activity.

254 These results suggested that the phenyl group can be modified but there are limitations to

255 the structural modifications that retain period-lengthening activity.

257 dichlorophenyl group on the pyrimidinedione (Fig. 3d). Phenyl (5a), p-fluorophenyl (5b),

p-tolyl (5e), p-methoxyphenyl (5f), $m$-bromophenyl (5i) o-fluorophenyl (5j), o-

259 chlorophenyl (5k), o-bromophenyl (5l), 2,4-difluorophenyl (5p), 2,5-dichlorophenyl (5r),

260 2,6-dichlorophenyl (5u), and 2,4,6-trichlorophenyl (5v) derivatives had no period-

261 lengthening activity. $p$-Bromophenyl (5c), $m$-fluorophenyl (5h), 3,4-difluorophenyl (5m),

262 3-chloro-4-fluorophenyl (5n), 2-fluoro-4-bromophenyl (5q), and 2,3-dichlorophenyl (5s)

263 had low period-lengthening activities (significant changes were noted only with the 25 or

$26450 \mu \mathrm{M}$ treatments). Reliable period-lengthening activities were found with the addition of $p$-iodophenyl (5d), p-trifluoromethyl (5g), and 3-chloro-4-methylphenyl (5o) (Fig. 3d)

266 analogues. Unexpectedly, period-shortening activity was found in the 2,4-dichlorophenyl

267 (5t, TU-923) modified small molecule (Fig. 3d).

Our SAR study indicated that both the pyrimidinedione and the pyrazole ring

269 were essential for period-lengthening activity (Fig. 4a,b). The phenyl group on the 
271 pyrimidinedione resulted in activity that shortened the circadian period. The period

272 shortening activity of TU-923 was further examined (Fig. 4c,d). We found that TU-923

273 shortened the period of both the morning (CCA1:LUC) and evening (TOC1:LUC) clock

274 reporters, confirming that $\mathbf{T U} \mathbf{- 9 2 3}$ shortens the clock period. TU-923 was also cytotoxic 275 at a higher concentration $(200 \mu \mathrm{M}$, Fig. S2).

276

277 TU-923 lengthens the circadian period in $\operatorname{prr}^{9}$ prr7 mutants

278 The effects of TU-892 and TU-923 on some clock-period mutants were also examined to 279 help determine the action mechanisms of these molecules. We hypothesized that mutants 280 impaired in a crucial gene associated with the mode of action of these molecules may 281 experience a decrease in period modulating activities when treated with these small 282 molecules. The short period mutants (ccal-1 lhy-11 double mutants, prr5-11 toc1-2 283 double mutants, or prr7-11 prr5-11 double mutants) and a long period mutant (prr9-10 284 prr7-11 double mutants) were treated with TU-892 or TU-923 (Fig. 5a). In the wild type, $25 \mu \mathrm{M}$ TU-892 and TU-923 lengthened and shortened the circadian period, respectively, 286 as described above. TU-892 lengthened the period in all of the clock mutants, indicating 287 that TU-892 does not require these genes for period lengthening. In contrast, the effect of 288 TU-923 diverged among the clock mutants. TU-923 shortened the period in the $c c a 1-1$ 289 lhy-12 and prr7-11 prr5-11 mutants, did not alter the period in prr5-11 tocl-2, and 290 lengthened the period in $\operatorname{prr} 9-10$ prr $7-11$. To validate the period-lengthening activity of 291 TU-923 in $\operatorname{prr} 9-10$ prr7-11, we analyzed the mutants during continuous treatment with different concentrations of TU-923 (Fig. 5b). The results indicated that TU-923 
293 lengthened the period in these mutants in a dose-dependent fashion. The period

294 lengthening effect of TU-923 in prr9-10 prr7-11 was about 4 - 8 h [Circadian Time (CT)-

295 corrected] at concentrations of 25 to $50 \mu \mathrm{M}$. These results suggested that the period

296 shortening activity of TU-923 is required for the functions of PRR 9 and $P R R 7$.

298 Discussion

By a combined approach using a large-scale phenotypic screening and a SAR

300 study, we found a period lengthening molecule, TU-892, and a period shortening 301 molecule, TU-923, both of which have a similar chemical structure. Our study clearly 302 indicated that $\mathbf{T U - 8 9 2}$ is not a CKL inhibitor, suggesting the presence of a new 303 pharmacologically tunable point for clock regulation. Importantly, reversing the direction 304 of period length has not been achieved by any of the small molecule analogues of kinase 305 inhibitors such as longdaysin and PHA767491 in animals and plants (Hirota et al., 2010; 306 Lee et al., 2019; Saito et al., 2019; Uehara et al., 2019). Longdaysin and PHA767491 307 were proposed to inhibit CK1 kinase by binding to the ATP-binding pocket. Generally, it 308 is quite difficult to make kinase activators that bind to the ATP-binding pocket, suggesting 309 that TU-892 and TU-923 are unlikely to be competitive inhibitors of ATP.

311 mammalian clock component cryptochrome, revealed a period shortening molecule 312 (Oshima et al., 2015). We speculated that TU-892 and its analogue TU-923 lengthens 313 and shortens the circadian period, respectively, by controlling critical components of the 314 Arabidopsis circadian clock. TU-892 and TU-923 structures differ only in the position of 
315 chloride in the dichlorophenyl moeity. 3,4-Dichlorophenyl can spin around on the bond

316 between the dichlorophenyl group and the pyrimidine in TU-892, whereas 2,4-

317 dichlorophenyl in TU-923 cannot spin due to steric hindrance of the 2-chloro

318 modification of the pyrimidine phenyl group. This structural difference may cause a

319 difference in affinity or preference for binding to target proteins. Insight into the structural

320 differences between TU-892 and TU-923 as well as the activity of TU-923 in prr9-10

321 prr7-11 will help reveal the full action mechanisms of these molecules for clock control.

Although the actual targets of TU-892 and TU-923 were not revealed in this

323 study, we propose a model for the action mechanisms of TU-892 and TU-923 in period

324 tuning (Fig. 5c). Due to their structural similarity, TU-892 and TU-923 likely target very

325 similar proteins or other endogenous factors $\left(X\right.$ and $X^{\prime}$ in Fig. 5c). The functions of $X$ and

$326 \mathrm{X}^{\prime}$ for period tuning are opposite. $P R R 9$ and $P R R 7$ participate in association with TU-923

327 and $\mathrm{X}^{\prime}$ or induce or activate $\mathrm{X}^{\prime}$. TU-923 becomes bound to $\mathrm{X}$ if $P R R 9$ and $P R R 7$ are

328 mutated. PRR5 and TOC1 are implicated downstream of TU-923 function, because the

329 period of TU-923 treatment in the prr5-11 toc1-2 mutant was similar to the control

330 experiment.

Since the plant clock regulates diverse biological processes including

332 photoperiodic flowering time regulation and the drought stress response, both regarded

333 as crucial traits for plant breeding programs, it should be possible to use clock modulators

334 as agrochemicals. Unfortunately, however, at high concentrations TU-892 and TU-923

335 also have harmful effects on seedling growth as well as period-changing activities (Figs.

336 S1, S2). Further derivatizations of TU-892 and TU-923 may provide molecules that will 
337 be useful as agrochemicals.

338

339 Acknowledgements

340 We thank Drs H. Kasahara (Tokyo University for Agriculture and Technology), M. Kubo

341 (Kumamoto University), and M. Hasebe (National Institute for Basic Biology, Okazaki,

342 Aichi, Japan) for providing the chemical library Hitfinder 10K, and Dr. A. Sato and Ms.

343 N. Kato for maintaining the TU-892 analogues. We thank Drs T. Kondo, K. Miwa, Y.

344 Kitayama, T. Matsuo, H. Shinohara, and Y. Matsubayashi (Nagoya University) for helpful

345 discussions. This work is supported in part by, a Grant-in-Aid for Scientific Research

346 (KAKENHI) from the Japan Society for the Promotion of Science (18H02136) to NN,

347 and a Grant-in-Aid for Scientific Research on Innovative Areas 18H04428 to J.Y., and

$34815 \mathrm{H} 05956$ to T.K. and $20 \mathrm{H} 05411$ to N.N. ITbM is supported by the World Premier

349 International Research Center (WPI) Initiative, Japan.

350

351 Author Contributions

352 TNU, ANS, EO, KI, and JY synthesized small molecules. ST conducted the chemical 353 screening. HM and NN analyzed circadian assays. AO analyzed in vitro CK1 assays. KI 354 and TK supervised the project. JY and NN conceptualized and wrote the paper.

\section{Competing interests}

357 The authors declare no competing interests. 
bioRxiv preprint doi: https://doi.org/10.1101/2020.05.25.113746; this version posted May 27,2020 . The copyright holder for this preprint

(which was not certified by peer review) is the author/funder, who has granted bioRxiv a license to display the preprint in perpetuity. It is made available under aCC-BY-ND 4.0 International license.

\section{Supporting Information}

360 Method: Synthesis Strategy for TU-892 and Analogues

361 Fig. S1 Treatment with a high concentration of TU-892 resulted in leaf bleaching.

362 Fig. S2 Treatment with a high concentration of TU-923 resulted in leaf bleaching. 
References

365

Bansagi T, Jr., Leda M, Toiya M, Zhabotinsky AM, Epstein IR. 2009. High-frequency oscillations in the Belousov-Zhabotinsky reaction. J Phys Chem A 113(19): 56445648.

Bargiello TA, Jackson FR, Young MW. 1984. Restoration of circadian behavioural rhythms by gene transfer in Drosophila. Nature 312(5996): 752-754.

Belbin FE, Hall GJ, Jackson AB, Schanschieff FE, Archibald G, Formstone C, Dodd AN. 2019. Plant circadian rhythms regulate the effectiveness of a glyphosatebased herbicide. Nat Commun 10(1): 3704.

de Montaigu A, Oeljeklaus J, Krahn JH, Suliman MNS, Halder V, de Ansorena E, Nickel S, Schlicht M, Plihal O, Kubiasova K, et al. 2017. The Root GrowthRegulating Brevicompanine Natural Products Modulate the Plant Circadian Clock. ACS Chem Biol 12(6): 1466-1471.

Dodd AN, Salathia N, Hall A, Kevei E, Toth R, Nagy F, Hibberd JM, Millar AJ, Webb AA. 2005. Plant circadian clocks increase photosynthesis, growth, survival, and competitive advantage. Science 309(5734): 630-633.

Goodspeed D, Chehab EW, Min-Venditti A, Braam J, Covington MF. 2012. Arabidopsis synchronizes jasmonate-mediated defense with insect circadian behavior. Proc Natl Acad Sci U S A 109(12): 4674-4677.

Harmer SL, Hogenesch JB, Straume M, Chang HS, Han B, Zhu T, Wang X, Kreps JA, Kay SA. 2000. Orchestrated transcription of key pathways in Arabidopsis by the circadian clock. Science 290(5499): 2110-2113.

Haydon MJ, Mielczarek O, Robertson FC, Hubbard KE, Webb AA. 2013. Photosynthetic entrainment of the Arabidopsis thaliana circadian clock. Nature 502(7473): 689-692.

Hirota T, Lee JW, Lewis WG, Zhang EE, Breton G, Liu X, Garcia M, Peters EC, Etchegaray JP, Traver D, et al. 2010. High-throughput chemical screen identifies a novel potent modulator of cellular circadian rhythms and reveals CKIalpha as a clock regulatory kinase. PLoS Biol 8(12): e1000559.

Hirota T, Lee JW, St John PC, Sawa M, Iwaisako K, Noguchi T, Pongsawakul PY, Sonntag T, Welsh DK, Brenner DA, et al. 2012. Identification of small molecule 
activators of cryptochrome. Science 337(6098): 1094-1097.

Hirota T, Lewis WG, Liu AC, Lee JW, Schultz PG, Kay SA. 2008. A chemical biology approach reveals period shortening of the mammalian circadian clock by specific inhibition of GSK-3beta. Proc Natl Acad Sci U S A 105(52): 20746-20751.

Arabidopsis Genome Initiative. 2000. Analysis of the genome sequence of the flowering plant Arabidopsis thaliana. Nature 408(6814): 796-815.

Ishiura M, Kutsuna S, Aoki S, Iwasaki H, Andersson CR, Tanabe A, Golden SS, Johnson CH, Kondo T. 1998. Expression of a gene cluster kaiABC as a circadian feedback process in cyanobacteria. Science 281(5382): 1519-1523. ASSOCIATED1 in the Arabidopsis Circadian Clock. Plant Cell 28(3): 696-711.

Kondo T. 1990. Shortening of the Period of the Circadian-Rhythm by a K+ Channel Blocker, Tetraethylammonium, in the Duckweed Lemna-Gibba G3. Journal of Biological Rhythms 5(3): 187-194.

Lee JW, Hirota T, Ono D, Honma S, Honma K, Park K, Kay SA. 2019. Chemical Control of Mammalian Circadian Behavior through Dual Inhibition of Casein Kinase 1 alpha and delta. Journal of Medicinal Chemistry 62(4): 1989-1998.

McClung CR. 2019. The Plant Circadian Oscillator. Biology (Basel) 8(1).

Millar AJ. 2016. The Intracellular Dynamics of Circadian Clocks Reach for the Light of Ecology and Evolution. Annu Rev Plant Biol 67: 595-618. nullifies the circadian clock in cyanobacteria through Hopf bifurcation. Proceedings of the National Academy of Sciences of the United States of America 114(22): 5641-5646. 


\section{Nakajima M, Imai K, Ito H, Nishiwaki T, Murayama Y, Iwasaki H, Oyama T, Kondo} T. 2005. Reconstitution of circadian oscillation of cyanobacterial KaiC phosphorylation in vitro. Science 308(5720): 414-415.

Nakamichi N. 2015. Adaptation to the local environment by modifications of the photoperiod response in crops. Plant Cell Physiol 56(4): 594-604.

Nakamichi N, Kita M, Ito S, Yamashino T, Mizuno T. 2005. PSEUDO-RESPONSE REGULATORS, PRR9, PRR7 and PRR5, together play essential roles close to the circadian clock of Arabidopsis thaliana. Plant Cell Physiol 46(5): 686-698.

Nohales MA, Kay SA. 2016. Molecular mechanisms at the core of the plant circadian oscillator. Nat Struct Mol Biol 23(12): 1061-1069.

Ono A, Sato A, Fujimoto KJ, Matsuo H, Yanai T, Kinoshita T, Nakamichi N. 2019. 3,4-Dibromo-7-Azaindole Modulates Arabidopsis Circadian Clock by Inhibiting Casein Kinase 1 Activity. Plant Cell Physiol 60(11): 2360-2368.

Oshima T, Niwa Y, Kuwata K, Srivastava A, Hyoda T, Tsuchiya Y, Kumagai M, Tsuyuguchi M, Tamaru T, Sugiyama A, et al. 2019. Cell-based screen identifies a new potent and highly selective CK2 inhibitor for modulation of circadian rhythms and cancer cell growth. Sci Adv 5(1): eaau9060.

Oshima T, Yamanaka I, Kumar A, Yamaguchi J, Nishiwaki-Ohkawa T, Muto K, Kawamura R, Hirota T, Yagita K, Irle S, et al. 2015. C-H Activation Generates Period-Shortening Molecules That Target Cryptochrome in the Mammalian Circadian Clock. Angewandte Chemie-International Edition 54(24): 7193-7197.

Panter PE, Muranaka T, Cuitun-Coronado D, Graham CA, Yochikawa A, Kudoh H, Dodd AN. 2019. Circadian Regulation of the Plant Transcriptome Under Natural Conditions. Front Genet 10: 1239.

Perales M, Mas P. 2007. A functional link between rhythmic changes in chromatin structure and the Arabidopsis biological clock. Plant Cell 19(7): 2111-2123.

Saito AN, Matsuo H, Kuwata K, Ono A, Kinoshita T, Yamaguchi J, Nakamichi N. 2019. Structure-function study of a novel inhibitor of the casein kinase 1 family in Arabidopsis thaliana. Plant Direct 3(9): e00172.

Salome PA, Weigel D, McClung CR. 2010. The role of the Arabidopsis morning loop components CCA1, LHY, PRR7, and PRR9 in temperature compensation. Plant Cell 22(11): 3650-3661.

Somers DE, Kim WY, Geng R. 2004. The F-box protein ZEITLUPE confers dosage- 

time. Plant Cell 16(3): 769-782.

Tamai TK, Nakane Y, Ota W, Kobayashi A, Ishiguro M, Kadofusa N, Ikegami K, Yagita K, Shigeyoshi Y, Sudo M, et al. 2018. Identification of circadian clock modulators from existing drugs. EMBO Mol Med 10(5). 1 is an actin-stabilizing small molecule identified from a chemical genetic screen for circadian clock effectors in Arabidopsis thaliana. Plant J 71(2): 338-352.

Uehara TN, Mizutani Y, Kuwata K, Hirota T, Sato A, Mizoi J, Takao S, Matsuo H, the Arabidopsis circadian clock. Proc Natl Acad Sci U S A 116(23): 11528-11536.

Yanovsky MJ, Kay SA. 2002. Molecular basis of seasonal time measurement in Arabidopsis. Nature 419(6904): 308-312.

Yerushalmi S, Yakir E, Green RM. 2011. Circadian clocks and adaptation in Arabidopsis. Mol Ecol 20: 1155-1165. 
(a)

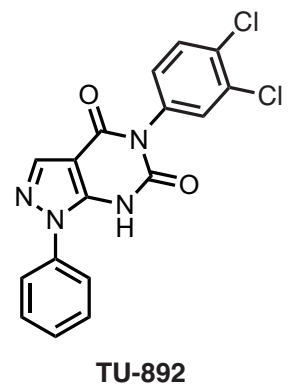

(b)

CCA1:LUC

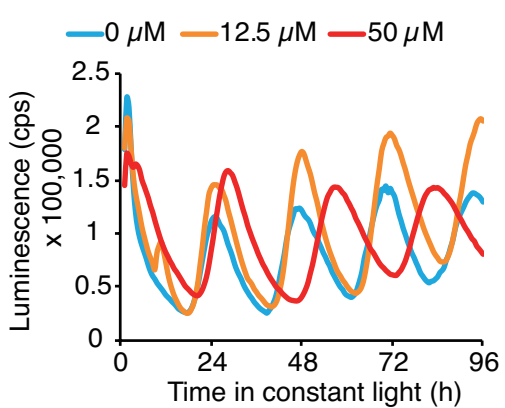

(d)

TOC1::LUC

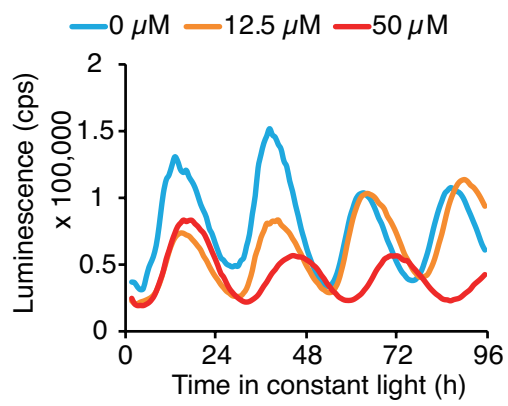

(c) CCA1:LUC

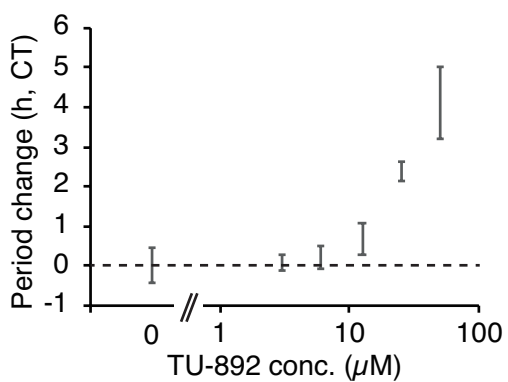

(e)

TOC1::LUC

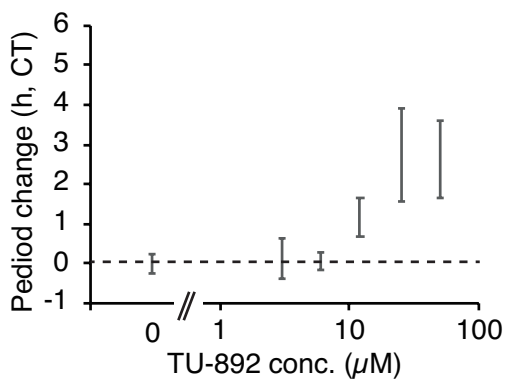

\section{Figure 1}

481 TU-892 lengthens the Arabidopsis circadian period. (a) Chemical structure of TU-892 482 (5-(3,4-dichlorophenyl)-1-phenyl-1,7- dihydro-4H-pyrazolo[3,4- $d$ ]pyrimidine-4,6(5H)483 dione). Circadian luciferase reporter activity in Arabidopsis treated with TU-892 $484 C C A 1: L U C$ (b), and TOC1:LUC (d). Increases in period length relative to the untreated $485(0 \mu \mathrm{M})$ control indicate a dose-response (c) and (e) $(\mathrm{n}=7$ or 8 for each concentration, 486 with error bars indicating the standard deviation [S.D.]). 
(a)

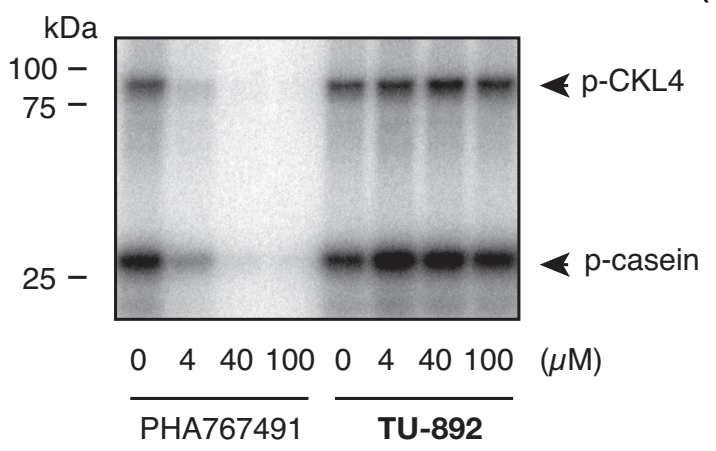

(c)

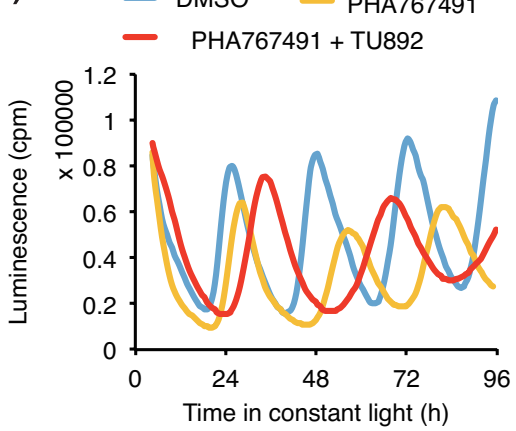

(b)

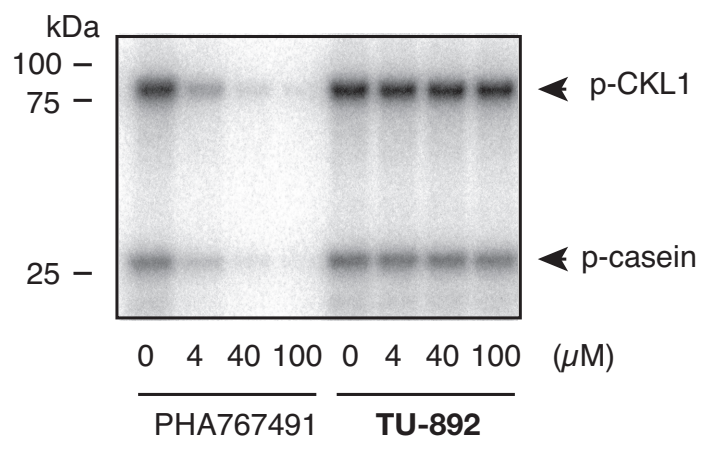

(d)

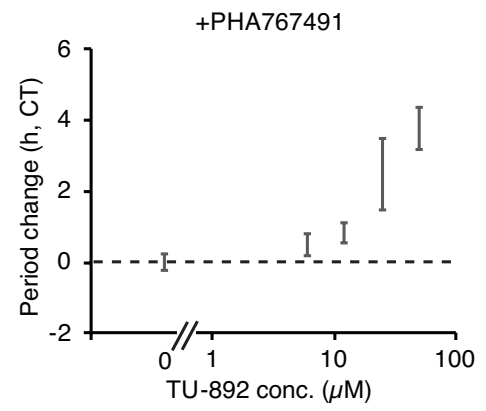

\section{Figure 2}

490 TU-892 is not a CKL inhibitor. Autoradiography of in vitro CKL4 (a) and CKL1 (b) 491 activities in the presence of TU-892. PHA767491 was used as a CKL inhibitor. (c) 492 Luminescence from circadian luciferase reporter CCA1:LUC activity in Arabidopsis 493 treated with $250 \mu \mathrm{M}$ PHA767491 and $25 \mu \mathrm{M}$ TU-892. (d) Increases in period length by 494 TU-892 relative to the untreated $(0 \mu \mathrm{M})$ control in the presence of $250 \mu \mathrm{M}$ PHA767491. 
(a)

(c)
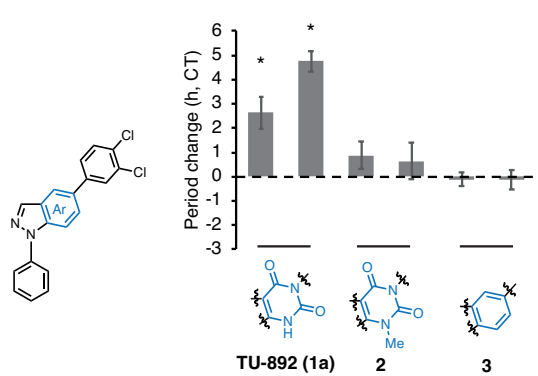

(b)

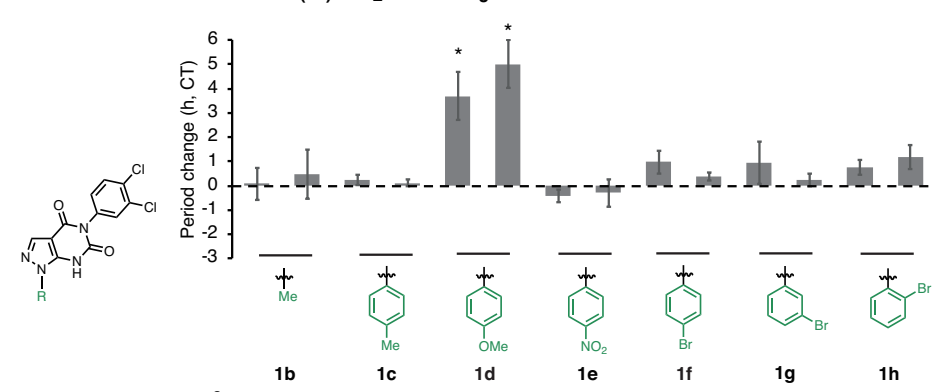

(d)
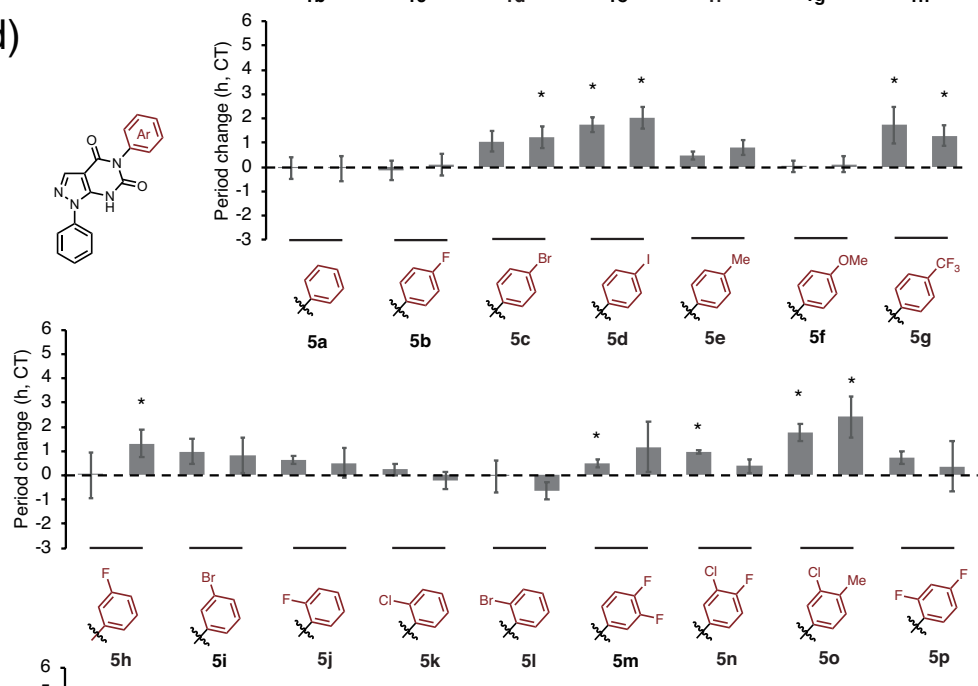

\section{Figure 3}

497 SAR study of TU-892. Circadian period changes after Arabidopsis seedling treatment 498 with 25 (left) or $50 \mu \mathrm{M}$ (right) TU-892 analogues substituted at the pyrimidinedione (a), 499 pyrazole (b), phenyl (c), or 3,4-dichlorophenyl (d) groups compared to solvent DMSO500 treated samples are shown $(n=4$, with S.D.). Asterisks indicate a significant change in 501 circadian period compared to that of DMSO-treated samples (Student's t-test $p<0.01$ ). 
(a)

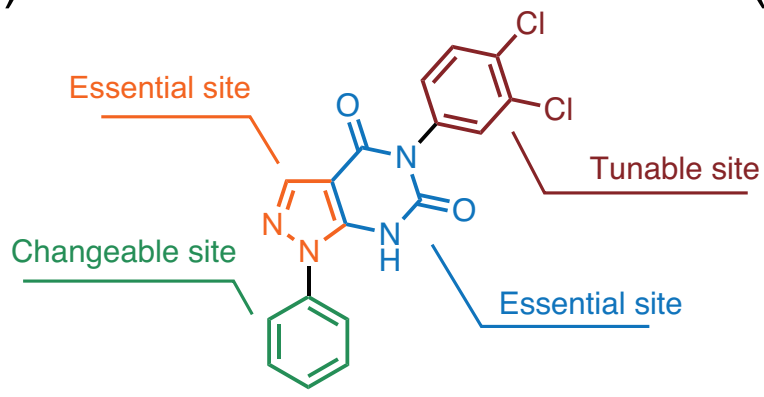

(c)

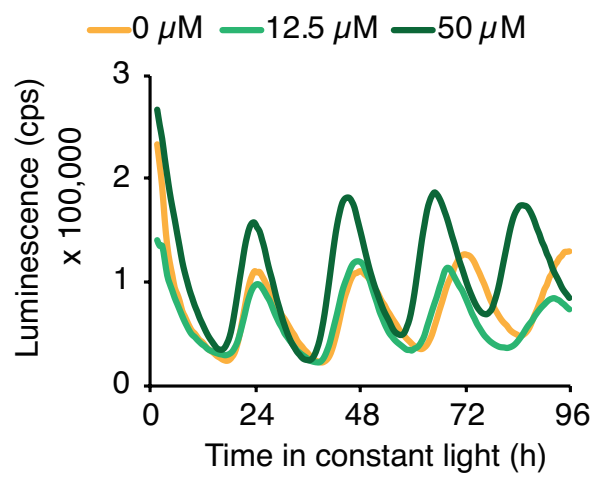

(e)

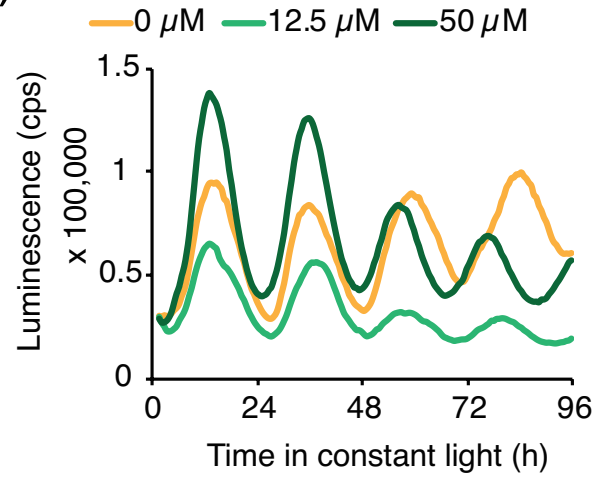

(b)

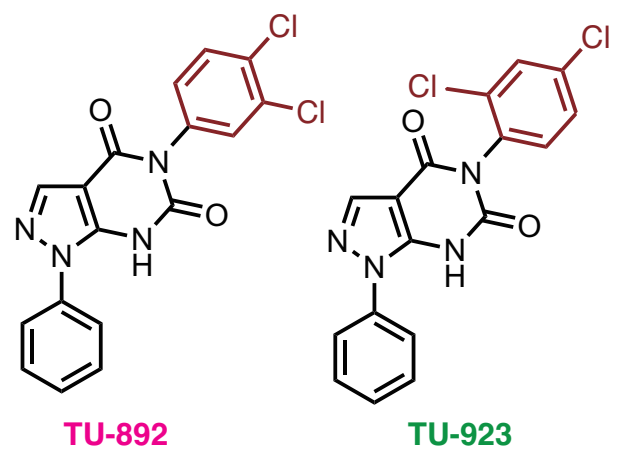

(d)

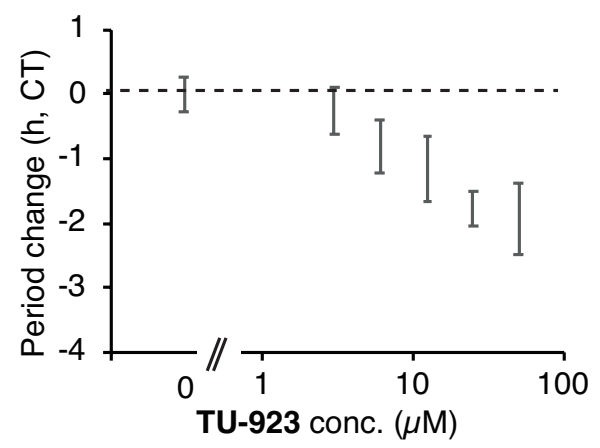

(f)

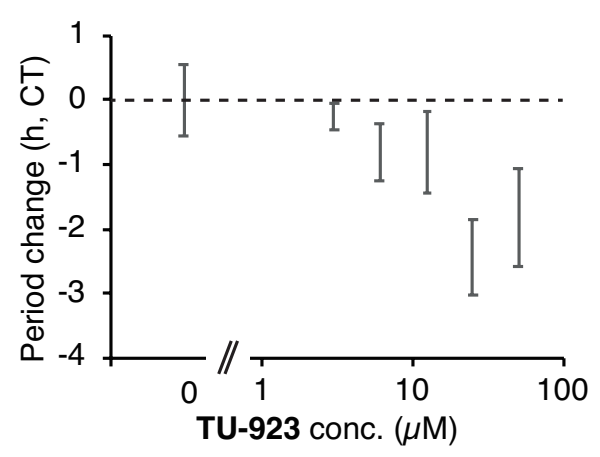

\section{Figure 4}

505 TU-923 shortens the circadian period. (a) Summary of results from a SAR study of TU-

506 892. (b) Structures of TU-892 and TU-923 (5-(2,4-dichlorophenyl)-1-phenyl-1,7-

507 dihydro-4H-pyrazolo[3,4- $d]$ pyrimidine-4,6(5H)-dione). Luminescence of circadian

508 luciferase reporters CCA1:LUC (c) or TOC1:LUC (d) in Arabidopsis treated with TU509 923. Period length changes relative to the untreated $(0 \mu \mathrm{M})$ controls of CCA1:LUC (e) or 510 TOC1:LUC (f) ( $\mathrm{n}=5$ to 8 for each concentration, with error bars for S.D.). 
(a)
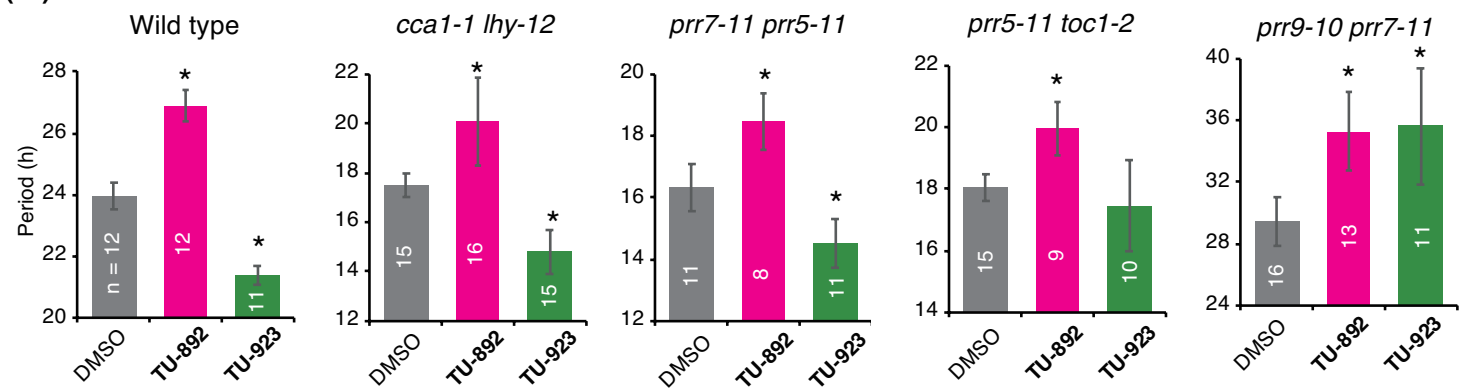

(b)

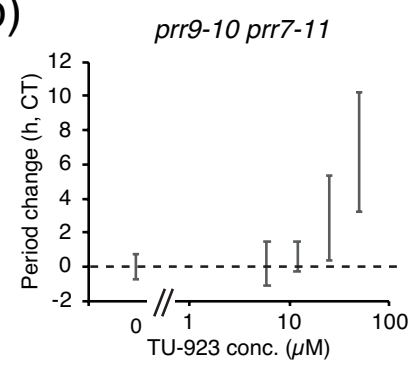

(c)

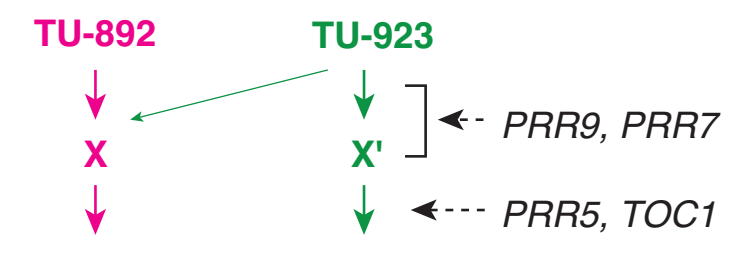

Lengthening Shortening

\section{Figure 5}

514 Effect of TU-892 and TU-923 on clock period mutants. (a) Effect of $25 \mu \mathrm{M}$ TU-892 and

515 TU-923 on period length of the ccal-1 lhy-12, prr7-11 prr5-11, prr5-11 tocl-2, and prr9-

51610 prr7-11 mutants. Asterisks indicate significant differences in period length compared 517 to that of the control (DMSO treatment) (Tukey-HSD test $p<0.05$ ). Error bars indicate 518 the S.D. (b) Period length change due to TU-923 treatment relative to the untreated (0 $519 \mu \mathrm{M})$ control of $p r r 9-10$ prr7-11 ( $\mathrm{n}=5-11$, with error bars for S.D.). All experiments were 520 performed twice with similar results. (c) Proposed action mechanism for TU-892 and 521 TU-923. TU-892 and TU-923 target $\mathrm{X}$ and $\mathrm{X}^{\prime}$ (an $\mathrm{X}$ homologue), respectively. $P R R 9$ and $522 \quad P R R 7$ participate in the interaction between TU-923 and $\mathrm{X}^{\prime}$ or induce or activate $\mathrm{X}^{\prime}$. TU523923 may change the target to $\mathrm{X}$, if $P R R 9$ and $P R R 7$ are mutated. PRR5 and TOC1 are 524 possibly implicated downstream of X'. 


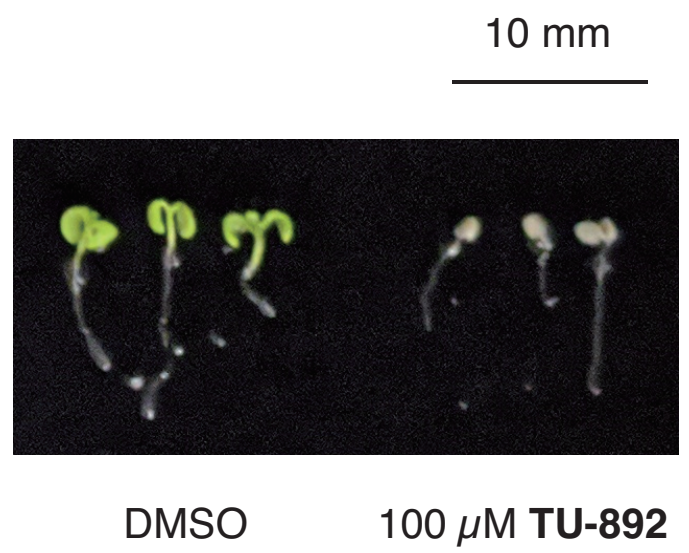

526

527 Fig. S1 Treatment with a high concentration of TU-892 resulted in leaf bleaching.

528 Individual seedlings in a well of a 96-well plate were treated with $100 \mu \mathrm{M}$ TU-892 for 1

529 week. Cotyledons were bleached by the treatment.

530

531

533 Fig. S2 Treatment with a high concentration of TU-923 resulted in leaf bleaching.

534 Individual seedlings in a well of a 96-well plate were treated with $200 \mu$ M TU-923 for 1

535 week. Cotyledons were bleached by the treatment.

536 